\title{
A catering economic survey of steamed dishes in Wuhan based on big data of Meituan application
}

\author{
Li Huihui ${ }^{1}$, Wang Jing ${ }^{1}$, Zhen Shuang ${ }^{1}$, He Siyun ${ }^{1 *}$ \\ ${ }^{1}$ College of Food Technology, Institute of Mianyang three Steamed dishes in China Wuhan Business University Wuhan, China
}

\begin{abstract}
The origin, quantities distribution and different market positioning of steamed dishes stores in Wuhan were analyzed based on the big data of Meituan application. By April 2020, there were a total of 230 steamed dishes restaurants in Wuhan, mainly distributed in Hongshan district (63), Jianghan district (47) and Wuchang district (35). Now the largest steamed dishes restaurant chains was "Zhen Kungfu" originated from Cantonese cuisine while "Liuyang steamed dishes" and "Zhen Liuji" restaurants were in the second place which came from "Home of steamed dishes in China". All of them were rapidly expanding in the form of Chinese fast-food chains in recent years and located either near the business district or the airport/station. On the contrary, "Mianyang three streamed dishes" restaurants with regional characteristics was few and scattered. It had not yet formed a scale with its unclear product positioning. A survey of consumers' preference showed that about $22 \%$ of consumers chose steamed dishes when eating out. With the increase of age, more and more people would opt for steamed dishes. Half of consumers over 40 chose steamed dishes. On the other hand, there were double peaked at 20 Yuan and 45 Yuan per person in consumers' psychological prices. These results would provide reference for the development of "Mianyang three streamed dishes" and Wuhan catering economy.
\end{abstract}

\section{Introduction}

Using water vapor as heat transfer medium, "Steaming" makes food raw materials matured ${ }^{[1]}$. Since its inception in the late Neolithic period, the steaming method had been constantly developed and improved. By the time $Q i$ Min Yao Shu was written in the Northern Wei Dynasty, the main steaming techniques and various Steamed utensils had been basically mature and passed down to this day ${ }^{[2]}$. Compared with the high temperature of $300^{\circ} \mathrm{C}$ for frying, the temperature of steaming was around $100^{\circ} \mathrm{C}$, so the loss of thermal decomposition and oxidation of nutrients in food was less, and no harmful substances were produced ${ }^{[3]}$. Steaming was a healthy way of cooking. In recent years, the combination of steamed dishes and central kitchen had led to the standardization, scale and industrialization of steamed dishes and gradually formed Chinese fast food of steamed dishes ${ }^{[4]}$, which had great potential for development. In order to understand the current situation of steamed dishes in Wuhan market, this paper analyzed the number and distribution of steamed dishes stores in Wuhan based on the big data of Meituan platform. These results would provide reference for the development of "Mianyang three streamed dishes" and Wuhan catering economy.

\section{Data acquisition and research method}

\subsection{Data sources}

The quantities distribution, dish price and other data of steamed dishes stores in Wuhan were from network platform. "Meituan.com" and "Dianping.com" these two apps with the highest market share were compared. Because the data of Dianping was incomplete and scattered, Meituan was chose as data source. In addition, the number and distribution of the restaurants were verified by "Amap.com" to ensure the credibility of the data. Questionnaires about consumers' preferences for steamed dishes were made and released through "wjx.cn".

\subsection{Data processing, statistics and analysis}

Stores closed or temporarily closed due to decoration or the pandemic of COVID-19 were not included in the number and distribution of stores in the statistics. The data obtained from the Meituan website were counted and plotted by Excel. A total of 168 questionnaires were distributed and 166 were recovered, with an effective rate of $98.8 \%$. 


\section{Results and analysis}

\subsection{The number and distribution of steamed dishes stores in Wuhan}

According to the statistics of Meituan website, there were a total of 230 steamed dishes restaurants in Wuhan by April 2020 as Table 1 showed. Among them, there were 179 stores in urban area while 51 restaurants in noncentral city. In central city area, the steamed dishes stores mainly distributed in Hongshan district (63), Jianghan district (47) and Wuchang district (35). These restaurants not only included 202 dishes stores, but also included 28 steamed soup stores.

Table1. The number and distribution of steamed dishes stores in Wuhan

\begin{tabular}{|c|c|c|c|c|c|c|c|c|c|}
\hline & HS & $\mathrm{JH}$ & WC & JA & QK & HY & QS & $\mathrm{NC}$ & total \\
\hline $\begin{array}{l}\text { Steamed } \\
\text { soup }\end{array}$ & 5 & 6 & 4 & 3 & 3 & & 3 & 4 & 28 \\
\hline $\begin{array}{l}\text { Zhen } \\
\text { Kungfu }\end{array}$ & 5 & 5 & 2 & 3 & & & & 3 & 18 \\
\hline $\begin{array}{l}\text { Liuyang } \\
\text { steamed } \\
\text { dishes }\end{array}$ & 9 & 1 & 1 & 1 & & 1 & & 3 & 16 \\
\hline Zhen Liuji & 3 & 5 & 2 & & & 1 & & & 11 \\
\hline $\begin{array}{l}\text { A simple } \\
\text { diet steam } \\
\text { flesh }\end{array}$ & 2 & 2 & & 1 & & & & & 5 \\
\hline $\begin{array}{l}\text { Mianyang } \\
\text { three } \\
\text { streamed } \\
\text { dishes }\end{array}$ & 1 & 1 & & & & & 1 & 2 & 5 \\
\hline $\begin{array}{l}\text { Steamed } \\
\text { oysters } \\
\text { legend }\end{array}$ & 2 & 3 & & & & & & & 5 \\
\hline $\begin{array}{l}\text { Zhen } \\
\text { Ziwei }\end{array}$ & 2 & & 2 & & & & & & 4 \\
\hline $\begin{array}{l}\text { Zhen } \\
\text { Youwei }\end{array}$ & 1 & 1 & 1 & & & & & 1 & 4 \\
\hline $\begin{array}{l}\text { Zhen } \\
\text { Shanmei }\end{array}$ & 1 & & & & & 1 & & 1 & 3 \\
\hline $\begin{array}{l}\text { Steamed } \\
\text { seafood }\end{array}$ & & & 1 & & & & & 1 & 2 \\
\hline $\begin{array}{l}\text { Dong } \\
\text { yiwei }\end{array}$ & & & & & & 2 & & & 2 \\
\hline $\begin{array}{l}\text { Other } \\
\text { stores }\end{array}$ & 32 & 23 & 22 & 3 & 6 & 4 & 1 & 36 & 127 \\
\hline Total & 63 & 47 & 35 & 11 & 9 & 9 & 5 & 51 & 230 \\
\hline
\end{tabular}

Note: HS, Hongshan district; JH, Jianghan district; WC, Wuchang district; JA, Jiang'an district; QK, Qiaokou district; HY, Hanyang district; QS, Qingshan district; NC, Non-central districts.

Wuhan had a permanent resident population of 11.2 million and there were 230 steamed dishes stores by 2019 . On average, one steamed dishes restaurant served 49,000 people. Hongshan district and Jianghan district with a large number of steamed dishes stores, had 1.26 and 0.83 million permanent residents while they had 63 and 47 steamed dishes stores respectively, which meant a steamed dishes restaurant served 18000-20000 people in Hongshan district and Jianghan district. Even if steamed dishes stores in Hongshan and Jianghan district were close to saturation, other districts still had great potential for development. Of course, the distribution of these stores was not only directly related to the population of each district, but also closely related to per capita GDP, which needed further study.

\subsection{Comparison of typical steamed dishes restaurants in Wuhan}

The data of Table 1 were obtained by searching keyword "steam" in Meituan application, which included 28 steamed soup stores. However "Jiangxi soup restaurant" was not included among them because its name was lack of "steam" although it really used this cooking method. So there were far more than 28 steamed soup restaurants in Wuhan. In the following article, we would focus on the steamed dishes restaurants in detail, and would not discuss the steamed soup stores.

Now the largest steamed dishes restaurant chains was "Zhen Kungfu" who had 18 branch while "Liuyang steamed dishes" and "Zhen Liuji" restaurants were in the second place which had 16 and 11 branch respectively in Wuhan's steamed dishes restaurants. The following were "A simple diet steam flesh" and "Mianyang three streamed dishes" restaurants which had 5 branch respectively. Both of them belonged to Hubei local cuisine, just as Figure.1 showed.

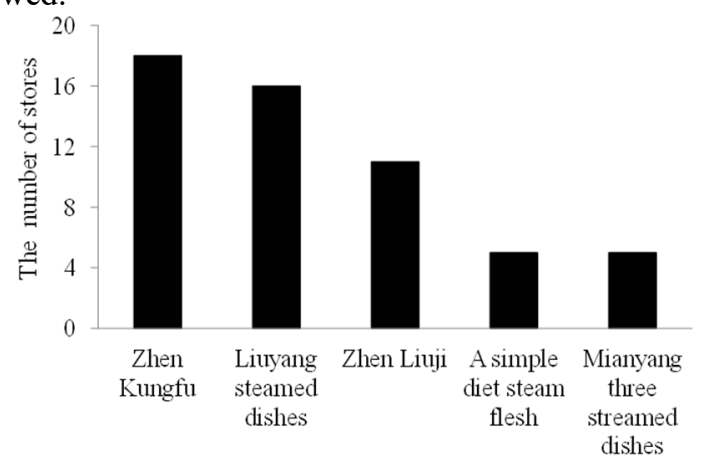

Fig 1. The number of typical steamed dishes stores in Wuhan

\subsubsection{Comparison of cuisines and dishes of typical steamed dishes restaurants in Wuhan}

"Zhen Kungfu" selling Chinese fast food was founded in Dongguan in 1990, whose brand positioning was"steaming is better for nutrition". In 2012, it was awarded the "Top 100 Catering Enterprises of the Year" by China Cuisine Association and ranked the number one in Chinese fast food. By 2019, Zhen Kungfu had more than 600 stores over the country including 18 branch stores in Wuhan. The menu of Zhen Kungfu was extremely varied from breakfast, snack, set meal, to soup. The dishes' taste also had the characteristics of Cantonese cuisine which tasted light and emphasized on the flavor of the original dish.

Both "Liuyang steamed dishes" and "Zhen Liuji" belonged to Hunan cuisine. Liuyang was awarded the title of "home of steamed dishes in China" in 2011 and Liuyang steamed dish was awarded the "China Geographical Symbol Collective trademark" in 2013. "Zhen Liuji" combined central kitchen and streamed dishes processing. There were more than 300 stores nationwide by 2019 with 
11 branch stores in Wuhan. The dishes of "Zhen Liuji" had obviously Hunan regional characteristics, with bacon and fermented black bean.

"A simple diet steam flesh" and "Mianyang three streamed dishes" belonged to Hubei local cuisine. "Mianyang three streamed dishes and its skill" was listed as provincial intangible cultural heritage by Hubei provincial government in 2010. In 2015, Xiantao was awarded the title of "Home of Mianyang three streamed dishes of China". In 2018,"Mianyang three streamed dishes" was named as one of the top ten famous dishes in Hubei province. "Mianyang three streamed dishes"restaurants in Wuhan were not chain stores, but the dishes were mostly steamed with rice flour, which reflected the strong local characteristics ${ }^{[5-6]}$.

\subsubsection{Comparison of distribution and site selection of typical steamed dishes restaurants in Wuhan}

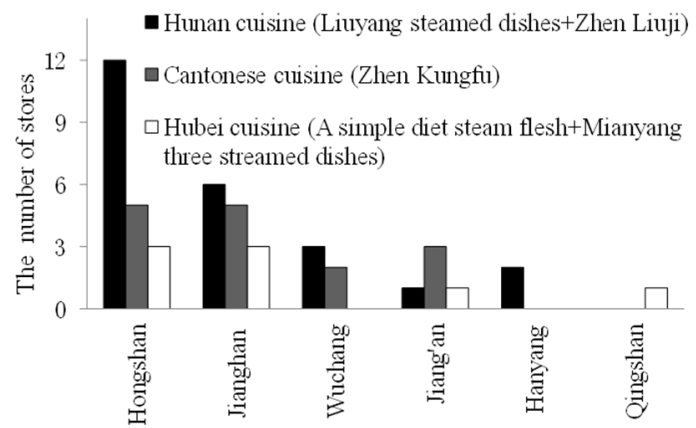

Fig 2. The distribution of typical steamed dishes stores in Wuhan

It could be seen from Figure. 2 that Hunan steamed dishes stores represented by "Liuyang steamed dishes" and "Zhen Liuji" were widespread in Hongshan, Jianghan, Wuchang, Jiang'an and Hanyang District, with a large overall scale and a total of 24 stores. "Zhen Kungfu" originated from Cantonese cuisine located in Hongshan, Jianghan, Wuchang and Jiang' an districts while "A simple diet steam flesh" and "Mianyang three streamed dishes" these two Hubei local restaurants only distributed in Hongshan, Jianghan, Jiang'an and Qingshan districts.

According to the information of Meituan and Amap, "Zhen Kungfu", "Zhen Liuji" and "Mianyang three streamed dishes" these three representative stores were selected to explore the siting strategies and business position (see Table 2 for details). Zhen Liuji had 5 stores located in Jianghan district and all of them located near office buildings, schools and hospitals in the business district. Taken the store at Wansongyuan Road as an example, there were two hospitals, three schools, a passenger terminal, a park and a large shopping mall within a radius of $1.5 \mathrm{~km}$. "Zhen Kungfu" site layout was different from "Zhen Liuji". It not only had restaurants near the business district, but also had six stores in Hankou, Wuchang, Wuhan railway stations and Tianhe airport. Zhen Kungfu had already spread to 11 airports and more than 60 railway stations all over the country. The station and airport had a large number of people, but the shop rent was also expensive. The operating costs were much higher than other place. Mianyang three streamed dishes in
Wuhan had few stores spread in Hongshan, Qingshan, Jiang' an and other non-central districts where had low rent. Although Mianyang three streamed dishes was well known in Wuhan, the brand effect had not yet formed while there had not a chain store.

\subsubsection{Comparison of unit price per customer of typical steamed dishes restaurants in Wuhan}

Both Zhen Kungfu and Zhen Liuji belong to Chinese style fast food. Consumer always chose set meal or 1-2 dishes every time. Zhen Kungfu used mantissa pricing to satisfy customers' desire for low price. The unit price per customer in station and airport was 30-45 Yuan,which is little higher than 20-25 Yuan in business district. The unit price per customer of Zhen Liuji was 20-30 Yuan in business district. Mianyang three streamed dishes was neither a chain store nor a fast food restaurant. There was no uniform specification for its dishes, so the unit price per customer had a great difference.

Table2. Comparison of typical steamed dishes restaurants in

\begin{tabular}{|l|l|l|l|}
\hline \multicolumn{2}{|c|}{ Zhen Kungfu } & Zhen Liuji & $\begin{array}{l}\text { Mianyang } \\
\text { three } \\
\text { streamed } \\
\text { dishes }\end{array}$ \\
\hline Cuisine & Cantonese cuisine & Hunan cuisine & $\begin{array}{l}\text { Hubei } \\
\text { cuisine }\end{array}$ \\
\hline $\begin{array}{l}\text { Site } \\
\text { selection }\end{array}$ & $\begin{array}{l}\text { Business district; } \\
\text { Station/airport }\end{array}$ & Business district & $\begin{array}{l}\text { Low-rent } \\
\text { place }\end{array}$ \\
\hline $\begin{array}{l}\text { Unit price } \\
\text { per customer }\end{array}$ & $\begin{array}{l}\text { 20-25 Yuan in } \\
\text { business district; } \\
30-45 \quad \text { Yuan } \\
\text { station/airport }\end{array}$ & $\begin{array}{l}\text { 20-25 Yuan in } \\
\text { business district }\end{array}$ & Diversity \\
\hline
\end{tabular}

\subsection{Consumer preference survey for steamed dishes}

\subsubsection{A brief introduction to the interviewees}

There were 166 respondents in this questionnaire, 52.4\% were male and $47.6 \%$ were female. $92.8 \%$ of the respondents were aged between 18 and 50, and 3.6\% were under 18 and over 50, respectively. Office staff account for $66.9 \%$ of the total, students $16.9 \%$, freelancers $10.2 \%$, and workers and other personnel $6.0 \%$. 91.0\% of the respondents were from Hubei province, with a smaller number from other provinces.

\subsubsection{Analysis of interviewees' preference for steamed dishes}

The consumer preference survey found that 47 percent of respondents chose stir-frying dishes when eating out while 22 percent chose steamed dishes and hot pot respectively. Besides $8 \%$ of respondents chose western food and $1 \%$ chose other options (see Figure.3). 


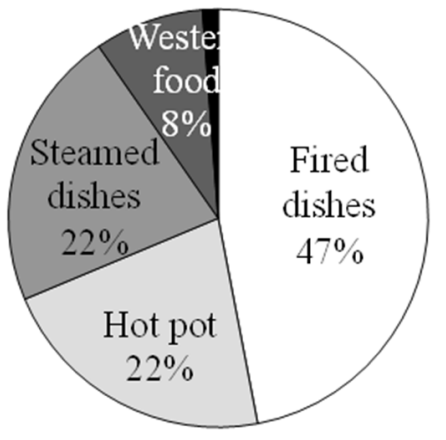

Fig 3. Consumer preference survey for steamed dishes

From the perspective of gender, men and women who chose steamed dishes for dining out account for about $21 \%$ of the total number, with little difference. But more men opted for stir-frying while women tended to prefer hot pot and western food, which showed that women paid more attention to lively atmosphere and dining environment. In terms of age, $60.0 \%$ of people aged $50-60,51.7 \%$ aged 41 $50,26.2 \%$ aged $31-40$ and $8.3 \%$ aged $18-30$ choose steamed dishes while 83.3 percent of young people under the age of 18 chose hot pot. It was obviously that with the growth of age, people were more and more inclined to choose steamed dishes and pay more attention to healthy diet.

\subsubsection{Analysis on the psychological price of steamed dishes by interviewees}

As could be seen from Figure. 4, the psychological price of steamed dishes reached two peaks, with 26.5 percent of respondents accepting a unit price of 20 Yuan per person and 23.5 percent of respondents accepting 45 Yuan. The occurrence of double peaks might be related to the different needs and expectations of consumers. Respondents who accepted a price of 20 Yuan per person might value the cheapness and convenience of steamed dishes more. However, respondents who accepted the price of 45 Yuan for each meal were more concerned about the nutrition of steamed dishes and willing to pay more for their health. Interestingly, the double peak of the respondents' psychological price for steamed dishes corresponded to the average price of a single customer of Zhen Kungfu in business district and the airport/station. This showed that pricing strategies of Zhen Kungfu was based on market research results, which was scientific and also accepted by the market.

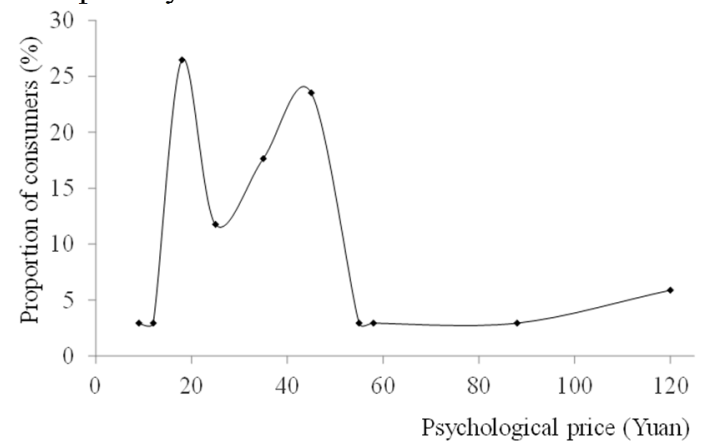

Fig 4. Psychological price of respondents on steamed dishes

\section{Conclusion and prospect}

Based on the big data of Meituan platform, this paper analyzed the number and distribution of steamed dishes stores in Wuhan. By April 2020, there were a total of 230 steamed dishes restaurants in Wuhan, including 179 stores in central districts and 51 restaurants in non-central districts. They were mainly distributed in Hongshan district (63), Jianghan district (47) and Wuchang district (35), which was not only directly related to the population of each district, but also closely related to per capita GDP, which needed further study. Now the largest steamed dishes restaurant chains was "Zhen Kungfu" originated from Cantonese cuisine. It had 18 branch stores. "Liuyang steamed dishes" and "Zhen Liuji" restaurants were in the second place which came from "Home of steamed dishes in China" with 16 and 11 branch stores respectively. All of them were rapidly expanding in the form of Chinese fastfood chains in recent years and located either near the business district or the airport/station. Although Mianyang three streamed dishes was well known in Wuhan with strong regional characteristics, the restaurants was few and scattered. It had not yet formed a scale with its unclear product positioning, and its development needed to learn from others. A survey of consumers' preference showed that about $22 \%$ of consumers chose steamed dishes when eating out. With the increase of age, more and more people would opt for steamed dishes, for example half of consumers over 40 age chose steamed dishes. There were great development potential in steamed dishes market. In addition, the psychological price of steamed dishes reached double peaks, with 26.5 percent of respondents accepting a unit price of 20 Yuan per person and 23.5 percent of respondents accepting 45 Yuan. The occurrence of double peaks might be related to the different needs and expectations of consumers. These results would provide reference for the development of "Mianyang three streamed dishes" and Wuhan catering economy.

\section{Acknowledgment}

Fund support: Comprehensive reform project of food quality and Safety Major of Wuhan Business University.

\section{REFERENCES}

1. Shi Qing, Chen Jiangpin. "The function and status of changshu steamed dishes in the banquet". Modern Food, Magn. Chin, vol. 2, pp. 9-15, 2019.

2. Wen Yi. "The origin of cooking technique 'steaming"'. J. Wuhan Busin. Univ., Magn. Chin, vol.30(4), pp. 4347, 2016.

3. Liu Xing, Wang Shengli, Wang Haibing. "Research on nutrition and development of steamed meat and vegetable powder". Food Techn. Econo., Magn. Chin, vol 40(2), pp. 66-68, 2015.

4. Sun Ruinan, Reng Xinping. "Research on the development trend of staple food industrialization and Chinese fast food industrialization". Modern Food, Magn. Chin, vol.2, pp. 54-55, 2020. 
5. Tian Mengyun, Xie Dingyuan. "Research and analysis of mianyang Three-steam market and thinking about its industrialization development". J Chuxiong Normal Univ., Magn. Chin, vol.34(1), pp.11-20, 2019.

6. Zhou Shenhong. "Origin and Development of 'Mianyang Three Steamed Dishes' ". J. Wuhan Busin. Univ., Magn. Chin, vol.29(5), pp. 29-34, 2015. 